\title{
EL VIVIR BIEN, UNA PROPUESTA DE LOS PUEBLOS INDÍGENAS A LA DISCUSIÓN SOBRE EL DESARROLLO
}

\section{LIVING WELL, A PROPOSAL FROM INDIGENOUS PEOPLE TO THE DEVELOPMENT DISCUSSION}

\author{
Elisa Canqui Mollo \\ Oficial de Programa IBIS en Bolivia \\ elisac@ibisur.org
}

Recibido: 02/03/2011

Aceptado: 26/05/2011

\begin{abstract}
Resumen
Últimamente hay una conciencia global sobre que la noción de desarrollo está pasando por serios procesos de crítica y reconstrucción, por las connotaciones evolucionistas y eurocéntricas predominantes en sus principales formulaciones, pensadas de cara a la construcción y ampliación de estados nacionales y de un proyecto económico y cultural dirigido a una progresiva modernización capitalista homogénea que busca la creciente satisfacción material e individual, fundada en formas que han amenazado con subordinar y transformar otros principios, culturas y conocimientos a su imagen y semejanza. Es en este marco, que el Vivir Bien, propuesta por los Pueblos Indígenas viene a sumarse a las distintas propuestas que han ido saliendo en cuanto a desarrollo se refiere. Esta propuesta comprendida por ellos como una traducción de las formas y estilos de vida, en su simplicidad muestra una compleja articulación entre la vida de los indígenas, sus recursos naturales y el vínculo estrecho entre ambos, dentro de un territorio.

Palabras Clave: Pueblos Indígenas, Vivir Bien, Desarrollo, territorio

Abstract

In recent times there is a global awareness of the fact that the notion of development is going through a process of criticism and reconstruction, due to the evolutionary and Eurocentric connotations in its formulation, which is oriented to the construction and expansion of nation-states and an economic
\end{abstract}


and cultural project led to a progressive and homogeneous capitalist modernization, aimed at increasing individual and material satisfaction. Those forms have threatened to subordinate and transform other principles, culture and knowledge. In this context we find the proposal of the indigenous peoples of Living Well. This proposal, understood by them as a translation of forms and lifestyles, shows in its simplicity, a complex articulation between the life of indigenous, their natural resources and the close link between them within a territory.

\section{UNA APROXIMACIÓN A LOS PUEBLOS INDÍGENAS}

\subsection{Pueblos indígenas, quiénes y cuántos}

Se estima que más de 300 millones de personas en el mundo son indígenas y pertenecen a los más de 5 mil Pueblos Indígenas en más de 70 países (ONU, 2010:37); los mismos se encuentran sistemáticamente marginados en casi todos los aspectos de la vida económica, social y política de estos países; pese a la riqueza económica, social, cultural y político-organizacional de los Pueblos Indígenas. Su distribución en el mundo va desde aquellos que aún se mantienen invisibles en las estadísticas de cada país, porque estos no los han reconocido como es el caso de la India, o son una mayoría como en el caso de Bolivia donde cerca del $67 \%$ de la población total es Indígena, viviendo en el áreas urbana ${ }^{1}$ y en el área rural.

Table 1: Pueblos Indígenas por Región (IWGIA, 2010:40) (millions)

China

South Asia

Former Soviet Union

Southeast Asia

South America

Africa

Central America/Mexico

Arabia

USA/Canada
105.23

94.90

0.40

29.84

19.53

21.98

19.07

15.41

3.29

\footnotetext{
${ }^{1}$ En el caso de Latinoamérica los censos de la ronda de 2000 revelan que alrededor de un $40 \%$ de la población indígena de la región reside en zonas urbanas, mientras que en el caso de la población no indígena esta cifra asciende a casi un $80 \%$. Fabiana Del Popolo, Ana María Oyarce y Bruno Ribotta. 2007. Indígenas urbanos en América Latina: algunos resultados censales y su relación con los Objetivos de Desarrollo del Milenio Notas de población n. ${ }^{\circ} 86 \bullet \mathrm{C}$ E PA Lp. 106
} 


$\begin{array}{lr}\text { Japan/Pacific Islands } & 0.00 \\ \text { Australia/New Zealand } & 0.46 \\ \text { Greenland/Scandinavia } & 0.10 \\ \text { Total } & 310.21\end{array}$

¿Qué entendemos por indígenas? No hay ninguna definición ampliamente aceptada de los pueblos indígenas. De hecho, el sistema de las Naciones Unidas no ha adoptado una definición de los pueblos indígenas, sino que ha desarrollado más bien una comprensión moderna de este término, basado en: autoidentificación con los pueblos indígenas a nivel individuo y aceptación como miembro por la comunidad; continuidad histórica con las sociedades precoloniales o pre-settler; fuerte vínculo con los territorios y los recursos naturales circundantes; distintos sistemas sociales, económicos o políticos; distinto idioma, cultura y creencias; formación de grupos no dominantes de la sociedad; y decisión de mantener y reproducir sus sistemas y entornos ancestrales como distintivos de los pueblos y comunidades (UNFPII, 2006: 3). Es así, que dentro del mundo académico, las políticas públicas y la cooperación internacional, la comprensión desarrollada en el informe de Martínez Cobo es la comúnmente utilizada, quien en su extenso texto señala:

Pueblos Indigenas son comunidades, pueblos y naciones indígenas los que, teniendo una continuidad histórica con las sociedades anteriores a la invasión y precoloniales que se desarrollaron en sus territorios, se consideran distintos a otros sectores de las sociedades que ahora prevalecen en esos territorios o en parte de ellos. Constituyen ahora sectores no dominantes de la sociedad y tienen la determinación de preservar, desarrollar y transmitir a futuras generaciones sus territorios ancestrales y su identidad étnica como base de su existencia continuada como pueblo, de acuerdo con sus propios patrones culturales, sus instituciones sociales y sus sistemas legales

Esa continuidad histórica puede consistir en la continuación, durante un período prolongado que llegue hasta el presente, de uno o más de los siguientes factores:

a) Ocupación de las tierras ancestrales o al menos de parte de ellas;

b) Ascendencia común con los ocupantes originales de esas tierras;

c) Cultura en general, o en ciertas manifestaciones especificas (tales como religión, vida bajo un sistema tribal, pertenencia a una comunidad indígena, trajes, medios de vida, estilo de vida, etc.);

d) Idioma (ya se utilice como lengua única, como lengua materna, como medio habitual de comunicación en el hogar o en la familia o como lengua principal, preferida, habitual, general o normal);

e) Residencia en ciertas partes del país o en ciertas regiones del mundo;

f) Otros factores pertinentes"

Aunque el término pueblo indígena ha prevalecido como un término genérico para muchos años, en algunos países puede haber una preferencia por otros tér- 
minos, como las tribus, primeras naciones, aborígenes, comunidades nativas, grupos étnicos, adivasi, janajati, originarios, etc. Términos indicativos de la ocupación y el hábitat, tales como cazadores-recolectores, nómadas, los campesinos, los pastores y gente de colina también existen y pueden utilizarse indistintamente con los pueblos indígenas.

\subsection{Los pueblos indígenas y el reconocimiento de sus derechos}

Los Pueblos Indígenas tradicionalmente vencieron una serie de dificultades; si bien la discriminación y el racismo continúan siendo problemas comunes, en el último siglo la globalización, el aumento de la población mundial y el cambio climático se han convertido en la base de la lucha y la demanda de los Pueblos Indígenas, tanto a nivel de local (en los países), como a nivel internacional. Cabe señalar que la globalización, el aumento de la población mundial y el cambio climático ejercen una significativa y preocupante presión en los territorios indígenas, donde tradicionalmente han desarrollado sus formas particulares de vida, y donde paradójicamente se encuentran los recursos naturales.

Esta lucha paulatinamente ha permitido su reconocimiento como "Pueblos" en dos de los principales instrumentos internacionales vigentes y ratificados, como son el Convenio $169(1989)^{2}$ y la Declaración de las Naciones Unidas sobre los Derechos de los Pueblos Indígenas $(2007)^{3}$. Los hitos más importantes del reconocimiento y la existencia de los Pueblos Indígenas y sus derechos, dentro del seno de las Naciones Unidas se traduce de la siguiente manera:

Mayo de 1971, el Consejo Económico y Social ECOSOC por resolución 1589(L) autoriza la preparación de un estudio del problema de la discriminación contra las poblaciones indígenas.

1982- se crea dentro de Naciones Unidas, en el ámbito de la Subcomisión para la Protección y Promoción de los Derechos Humanos, el Grupo de Trabajo sobre Poblaciones Indígenas (GTPI). El objetivo fue la elaboración de un Proyecto de Declaración de los Pueblos Indígenas, conformado por representantes de los Estados involucrados y por re-presentantes de los pueblos indígenas interesados.

1989- se aprueba el Convenio 169 sobre pueblos indígenas y tribales de la OIT por representantes de Gobiernos y de organizaciones de empleadores y trabajadores en la Conferencia Internacional del Trabajo de 1989. El Convenio

\footnotetext{
${ }^{2}$ Hasta Febrero del 2011, 22 países ha ratificado el Convenio 169, de los cuales, de los cuales una mayoría son países Latinoamericanos.

${ }^{3}$ El Estado Plurinacional de Bolivia es el único país que ha ratificado esta declaración mediante Ley No 3760 de 7 de noviembre de 2007, ratifica la Declaración de la Naciones Unidas sobre los Derechos de los Pueblos Indígenas, de 13 de septiembre de 2007.
} 
169 es el único instrumento jurídico internacional vigente dedicado específicamente a los Pueblos Indígenas. Es un Tratado Internacional de contenido obligatorio para los países firmantes que lo hayan ratificado.

10 Dic 1992, se proclama el año 1993 Año Internacional de los Pueblos Indígenas, lo que no contempla la resolución sobre esa conmemoración es conceder a los indígenas un escaño en la Asamblea General como reclamaron líderes.

21 Dic 1993 - con miras a fortalecer la cooperación internacional para la solución de los problemas a que se enfrentan los pueblos indígenas en esferas como los derechos humanos, el medio ambiente, el desarrollo, la educación y la salud.

23 Dic 1994 - la Asamblea general de las Naciones Unidas, decide declarar el 9 de agosto como Día internacional de los pueblos indígenas revitalizando los objetivos que se habían fijado en la resolución 48/163 del año 1993

En 1995 el GTPI aprueba por unanimidad el Proyecto de Declaración de los Pueblos Indígenas del Mundo, luego éste es aprobado por la Subcomisión para la protección y promoción de los Derechos Humanos, este proyecto hace referencia a los pueblos indígenas como sujetos de derecho pero no define el término.

En el año 2000 la Asamblea General aprobó la creación del FORO PERMANENTE SOBRE CUESTIONES INDÍGENAS. Ocho representantes indígenas en su calidad de "expertos independientes" son elegidos de acuerdo a un proceso de consultas en el ámbito nacional y regional y representan a los pueblos indígenas del mundo; y ocho expertos independientes denominados por los gobiernos. Durante el proceso para su establecimiento, se planteó que el nuevo órgano se denominará FORO PERMANENTE SOBRE LOS PUEBLOS INDÍGENAS, si bien esta recomendación no fue aceptada por los Estados.

En mayo de 2001, la Comisión de Derechos Humanos aprobó la designación de un Relator Especial de las Naciones Unidas sobre la situación de los derechos humanos y las libertades fundamentales de los Pueblos Indígenas.

Mayo 2002 - El Foro Permanente sostiene su primera reunión, en la sede de Naciones Unidas en New York, y hasta la fecha es una de las reuniones más grandes que el sistema tiene.

20 Dic 2004 La Asamblea proclamó el Segundo Decenio Internacional el 20 de diciembre de 2004, con la meta de continuar fortaleciendo la cooperación internacional en estas esferas, incluido el desarrollo económico y social (resolución 59/174).

13 de Sep. 2007, La Asamblea General de la Organización de las Naciones Unidas aprueba, tras dos décadas de negociaciones, la Declaración de las Naciones Unidas sobre los Derechos de los Pueblos Indígenas, con una abs- 
tención(Colombia) y cuatro votos en contra (Estados Unidos, Canadá, Australia y New Zelanda). Entre 2009-2010, estos países han cambiado de posición, siendo Estados Unidos el último país en adherirse a los postulados de esta Declaración el 16 de Diciembre del 2010.

14 de Dic. 2007, se establece un mecanismo de expertos sobre los derechos de los Pueblos Indígenas. Este mecanismo de expertos, órgano subsidiario del Consejo de Derechos Humanos, proporcionará al Consejo los conocimientos temáticos especializados sobre los derechos humanos de los pueblos (resolución 6/36).

Por otro lado desde el lado indígena, los Pueblos Indígenas y sus formas particulares de vida han sido la base fundamental para mantener su identidad como indígena; esto mismo les ha permitido constituirse en protectores y guardianes de los recursos de la biodiversidad, especialmente porque desde sus orígenes han desarrollado técnicas y mecanismos de coexistencia armónica con su entorno natural.

\section{EL DEBATE DEL DESARROLLO Y LOS PUEBLOS INDÍGENAS}

El concepto de desarrollo, desde la Segunda Guerra Mundial, a menudo se ha concebido en términos estrictamente económicos. El común denominador del objetivo se ha centrado en la búsqueda de crecimiento del Producto Interno Bruto (PIB) y la creencia general de que el desarrollo económico también se daría como desarrollo en otras esferas de la vida. El concepto de desarrollo se caracterizó como un proceso evolutivo que comenzó desde proveedores de los productos básicos, a través de la acumulación de capital a la industrialización, y éste a su vez conduce a la urbanización y la «modernización». Según teóricos liberales (Ramos, 2009:4), el producto final de desarrollo sería el establecimiento de las democracias meritocráticas con economías de mercado, la protección social y la desigualdad socioeconómica leve. Los teóricos marxistas fueron más allá y postulan sociedades igualitarias con propiedad colectiva de los medios de producción.

En este contexto, la posición y las particularidades de los pueblos indígenas fueron vistas siempre como contradictorias. Por un lado, marxistas y liberales teóricos consideran las sociedades de los pueblos indígenas como sociedades altamente móviles e igualitarias y sin embargo, al mismo tiempo, fueron considerados como sociedades 'crudas, primitivas e incivilizadas' donde su «desarrollo» se entiende como su asimilación en el "mundo civilizado" (Ramos, 2009: 4).

La comprensión actual de desarrollo implica la explotación de los recursos naturales donde se ve a los pueblos indígenas como 'obstáculos' al progreso, porque paradójicamente ocupan a menudo tierras que son ricas en recur- 
sos. No es sorprendente que la asimilación de los pueblos indígenas haya sido una estrategia para usurpar sus tierras y territorios, un paso necesario en el fomento del desarrollo ${ }^{4}$. En este contexto, el interés nacional siempre es precedido sobre los intereses de los pueblos indígenas. La mayor parte del siglo $\mathrm{XX}$, el trato otorgado a los pueblos indígenas fue muy similar a su tratamiento durante los siglos de colonialismo europeo y expansión (Hale, 2009:17).

Sin embargo desde la propuesta de los pueblos indígenas, traducida en críticas al desarrollo depredador, racista y discriminatorio y puramente económico, ha llevado y obligado de una u otra manera a seguir perfeccionando el concepto. Alternativas como «desarrollo humano», «desarrollo sostenible» $\mathrm{y}$ «enfoque al desarrollo basado en el de derechos humanos», han ido respondiendo a las preocupaciones y demandas de los Pueblos Indígenas; pero que no han repercutido más allá del discurso. Por ejemplo, dentro del sistema de Naciones Unidas, el Foro Permanente para las Cuestiones Indígenas repetidamente ha hecho hincapié en la necesidad de incorporar un enfoque basado en los derechos humanos para el cumplimiento de los Objetivos de Desarrollo de Milenio (ODM) que deben alcanzarse antes de 2015. Además, ha habido alguna sugerencia de, en el caso de «desarrollo sostenible», incluir a los Pueblos Indígenas como parte de la naturaleza.

Por otro lado, términos como Desarrollo con Identidad, Etnodesarrollo y Autodesarrollo son utilizados cuando se habla del "Desarrollo de los Pueblos Indígenas", sin que estas propuestas tampoco rescaten "el enfoque de desarrollo" de los pueblos indígenas que gira en torno a la cuestión de los derechos sobre la tierra y territorio, donde el territorio y los recursos no se reducen sólo como activos económicos, puesto que la tierra, el territorio y los recursos forman una base importante para la integridad social y cultural de los pueblos indígenas. Su vida misma.

Es sobre esta concepción y entendimiento del "desarrollo" que los pueblos indígenas están cada vez más gestionando y demandando la participación en los diálogos de política sobre cuestiones de desarrollo. Una clara posición es el cuestionamiento sobre la lógica del desarrollo, particularmente en grandes proyectos de infraestructura en sus tierras, tales como las industrias extractivas, carreteras o presas, que no sólo ha generado y agudizado la pobreza y la desigualdad severa, sino que también ha alimentado otros problemas sociales tales como la desintegración de las familias, la vida en la

${ }^{4}$ Estas estrategias también estuvieron acompañadas por políticas ambientalistas, como el de las áreas protegidas, mecanismo que permitió la usurpación de territorios indígenas en nombre de la conservación, sin considerar que la existencia de estos reservorios naturales era gracias a la visión holística de manejo del territorio. 
comunidad, el alcoholismo y el suicidio entre los jóvenes. La propuesta indígena, en cuanto a este ejemplo en particular se refiere, es que se continúe con los proyectos, pero siempre y cuando éstos respeten los derechos de la autodeterminación de los Pueblos Indígenas, es decir, que los proyectos deberán fortalecer las capacidades de gestión, conservación y aprovechamiento de los recursos que existen dentro de estos territorios; y en caso de que se afecte parte del territorio para construcción de estas infraestructuras, no es válida una simple indemnización, sino que ésta deberá comprender una restitución del territorio en las mismas condiciones.

En esta misma línea Amartya Sen, (Perez de Cuellar, 1996:31) ha hecho notar, que el binomio cultura-desarrollo se ha vuelto un imperativo de reflexión no sólo para los pueblos indígenas sino para el conjunto de las sociedades: "Un país no se identifica necesariamente con una sola cultura. Muchos países, tal vez la mayoría, son multiculturales, multinacionales y multiétnicos..." Y agrega: es indispensable reconocer el papel instrumental muy extendido de la cultura en el desarrollo y admitir al mismo tiempo que este papel no agota todo lo que hay de cultural en la apreciación del desarrollo. La cultura desempeña igualmente un papel porque es un fin deseable en sí mismo, porque da un sentido a nuestra existencia [...] Sin embargo, cuando se trata de la cuestión esencial de saber por qué concentrarse en estos objetivos particulares (entre los cuales se encuentra el crecimiento económico, la reducción de las desigualdades, la conservación del ambiente, etcétera), la cultura se debe entender de una manera más fundamental -no como un instrumento al servicio de tales o cuales fines, sino como la base social de los fines mismos. No podemos comprender la denominada dimensión cultural del desarrollo sin tomar conciencia de cada uno de estos dos papeles de la cultura".

\subsection{Pueblos indígenas: etnodesarrollo, autodesarrollo, desarrollo con identidad.}

El concepto de los pueblos indígenas de "desarrollo" adopta un enfoque integral que incluye sus aspiraciones, el respeto y la protección de su diversidad y singularidad. Los pueblos indígenas desean convertirse en agentes de su propio desarrollo y tienen la visión de promover un paradigma de desarrollo diferente al actual, como se establece en los artículos 3 y 32 de la declaración de las Naciones Unidas sobre los derechos de los pueblos indígenas. El paradigma de desarrollo de los pueblos indígenas podría basarse en los derechos colectivos y la ética de participación en los beneficios, afirmando su conexión importante a sus tierras y territorios, y capaz de hacer frente a los retos actuales. Un paradigma indígena podría tener una visión global que incluye el cre- 
cimiento económico, el aprovechamiento y conservación sostenible del medio ambiente y la afirmación de los derechos sociales, económicos y culturales de los pueblos indígenas.

Frente a esta realidad de los Pueblos Indígenas, académicos y especialistas en el tema han realizado algunas aproximaciones al desarrollo que correspondería a los pueblos indígenas, y que de una u otra manera reflejen la realidad y las particularidades de los pueblos indígenas.

Etnodesarrollo, entendido como la ampliación y consolidación de los ámbitos de cultura propia, mediante el fortalecimiento de la capacidad autónoma de decisión de una sociedad culturalmente diferenciada para guiar su propio desarrollo y el ejercicio de la autodeterminación, cualquiera que sea el nivel que considere, e implican una organización equitativa y propia del poder. Esto significa que el grupo étnico es unidad político-administrativa con autoridad sobre su propio territorio y capacidad de decisión en los ámbitos que constituyen su proyecto de desarrollo dentro de un proceso de creciente autonomía y autogestión (Bonfil, 1995: 47). Este término, etnodesarrollo si bien recibió aceptación en la marco de la cooperación internacional, e inclusive por los Estados, no paso lo mismo a nivel de los Pueblos Indígenas.

Autodesarrollo, conceptualizado como la elaboración, aplicación y proyección de los propios modelos y concepciones de desarrollo de los Pueblos Indígenas, definidos desde sus respectivas identidades diferenciadas, en orden a satisfacer adecuadamente sus necesidades individuales y colectivas; este derecho al autodesarrollo conllevaría al reconocimientos de los sistemas particulares de organización de los pueblos indígenas, incluyendo aspectos tales como la educación, la medicina, los sistemas de justicia o de resolución de conflictos (AECID, 2008: 18.), concepto que para los pueblos indígenas se refiere a los seres humanos y no a las cosas. En los pueblos indígenas la producción de bienes y el consumo dan paso a otras actividades que tienen que ver con el arte, con el ocio, con la espiritualidad, y en ese orden de ideas las opciones de autodesarrollo de los pueblos indígenas se juzgan en la medida en que cualitativamente mejoren la calidad de vida de todas las personas y de la persona toda, en un clima de armonía constante con la naturaleza.

Otro término que ha ido generando espacios de discusión y respuestas a la búsqueda de un concepto es el Desarrollo con Identidad, concebido como la transformación integral, auto y cogestionaria, de las condiciones de existencia social, económica, política, y cultural con que los pueblos indígenas y originarios se relacionan entre sí, con la naturaleza y con la sociedad nacional, de acuerdo con sus usos y costumbres, en condiciones de dignidad, equidad, oportunidad e institucionalidad para su desarrollo integral (MACPIO, 1998: 8). 
Entre 2004 y 2005, el Banco Interamericano de Desarrollo (BID), en el marco del proceso de elaboración de la Política y la Estrategia para Pueblos Indígenas, desarrolló un concepto que es la base de la propuesta de la política y la estrategia. Este concepto fue construido en un proceso de consulta con la participación de las organizaciones indígenas de la región y el consejo asesor indígena. En este proceso, principalmente en el espacio del Consejo Asesor Indígena, quienes acompañaban el trabajo del equipo del BID, responsable de la elaboración de ambos instrumentos, propusieron cambiar el objetivo de la política, que debería estar orientado a buscar el Vivir Bien de los Pueblos Indígenas y no al Desarrollo con Identidad.

El producto de este proceso, y el concepto en el que se basa tanto la política como la estrategia, entiende el Desarrollo con Identidad como un proceso que comprende el fortalecimiento de los pueblos indígenas, la armonía con el medio ambiente, la buena administración de los territorios y recursos naturales, la generación y el ejercicio de autoridad, y el respeto a los valores y derechos indígenas, incluyendo derechos culturales, económicos, sociales e institucionales de los pueblos indígenas, de acuerdo a su propia cosmovisión y gobernabilidad. Este concepto se sustenta en los principios de equidad, integralidad, reciprocidad y solidaridad y busca consolidar las condiciones en que los pueblos indígenas y sus integrantes puedan contar con bienestar y crecer en armonía con su entorno, aprovechando para ello, según sus propias prioridades, el potencial de sus bienes culturales, naturales y sociales (BID, 2006: 4).

Continuando con este debate, el Foro Permanente para las Cuestiones Indígenas de las Naciones Unidas en su informe de la novena sesión, cuyo tema principal fue "Los pueblos indígenas: desarrollo con cultura e identidad: artículos 3 y 32 de la Declaración de las Naciones Unidas sobre los derechos de los pueblos indígenas" llevado a cabo entre el 19 a 30 de abril de 2010, señala que los conceptos de los pueblos indígenas sobre el desarrollo con cultura e identidad se caracterizan por un enfoque holístico, que procura cimentarse en los derechos y la seguridad de la colectividad y en un mayor control y gobierno autónomo de las tierras, los territorios y los recursos. Esos conceptos se basan en la tradición y el respeto a los antepasados, pero también están proyectados hacia el futuro. Se basan en una filosofía restaurativa sustentada en los valores de reciprocidad, solidaridad, equilibrio, sostenibilidad, intercambio y colectividad. Y recomienda que los Estados, el sistema de las Naciones Unidas y otras organizaciones intergubernamentales proporcionen apoyo político, institucional y financiero, de conformidad con el artículo 42 de la Declaración de las Naciones Unidas sobre los derechos de los pueblos indígenas, a los esfuerzos de los pueblos indígenas, de manera que puedan consolidar sus propios modelos y conceptos de desarrollo y 
prácticas del buen vivir (por ejemplo, sumak kawsay, suma qamaña, laman laka, gawis ay biag), que se sustentan en las cosmologías, filosofías, valores, culturas e identidades indígenas, y que coordinen los esfuerzos por aplicar la Declaración (UNPFII, 2010: 3).

\section{EL VIVIR BIEN Y LOS PUEBLOS INDÍGENAS}

Etnodesarrollo, Autodesarrollo, Desarrollo con Identidad, son términos utilizados con el propósito de traducir el concepto de desarrollo para los Pueblos Indígenas, sin que estas aproximaciones reflejen realmente el significado holístico que implica el mismo, y que además traduzca en una palabra una forma particular de vida que implica, más allá de la persona indígena, también la vida dentro el territorio: tan importante es un árbol, una montaña, un río como un animal; pero también está la articulación y vinculación entre cada uno de estos elementos del territorio que conforman binomios y trinomios que hacen la salud, la educación y la gestión de los recursos naturales. Es en este marco en el que se han basado las críticas constantes de los Pueblos Indígenas hacia los modelos de desarrollo, la crisis económica y ambiental de los últimos años, que ha hecho posible que los especialistas en el tema realicen esfuerzos para conceptualizar y encontrar un término apropiado que refleje esta propuesta que viene de la propia vivencia de los Pueblos Indígenas y su concepción del desarrollo.

El Vivir Bien, ¿es algo nuevo? Es un intento más de traducir esta realidad particular de vida de los Pueblos Indígenas con su entorno en un concepto; ¿o es que, por primera vez, la voz y propuesta indígena es tomada en cuenta y desmenuzada en los medios académicos, de especialistas y de tomadores de decisión, que la analizan y buscan una respuesta y explicación técnica? Definitivamente es la segunda alternativa. Hasta ahora muchas de las propuestas de los Pueblos Indígenas no han sido consideradas como tal, más allá del proceso de discriminación histórica. Sin embargo, pareciese que la situación actual que vive el planeta está dando razón, y es quizá en este marco que esta propuesta de los Pueblos Indígenas, el Vivir Bien, pueda coadyuvar a la definición de políticas públicas en aquellos países, regiones, gobernaciones, municipios y comunidades con población indígena significativa con respecto a los no indígenas.

Al realizar un ejercicio de traducir el término "desarrollo" en las lenguas indígenas, uno se encuentra con que no existe un término que traduzca y se aproxima al significado, tal como se entiende en el español, (cabe señalar que con ello no estamos señalando que el Vivir Bien es igual a Desarrollo).

En muchas lenguas indígenas, El Vivir Bien, o Bien Vivir (palabra compuesta) se aproxima a lo que en español entendemos por "Desarrollo", pero 
no necesariamente igual. El Vivir Bien, tiene una significado más allá de una simplicidad, y refleja la visión holística con que los Pueblos Indígenas ven el mundo y la vida; dentro de la cual pareciese que se encuentran elementos comunes como la armonía con la naturaleza, la identidad, la espiritualidad o la reciprocidad, entre otros.

Ngobe (Panama): Ti nûle kûin: estar bien, con salud, sin preocupación, sentirse feliz. Tener una buena relación con la naturaleza ${ }^{5}$.

Aymara (Bolivia, Chile, Perú) "suma qamaña" vivir bien, vivir en plenitud, estar bien. (Huanacuni, 2009: 56) amplía esta traducción como "vivir en armonía y equilibrio; en armonía con los ciclos de la Madre Tierra, del cosmos, de la vida y de la historia, y en equilibrio con toda forma de existencia".

Guarani-Bolivia-Paraguay, Argentina, Brasil: "Ñandereko" comprensión de buena vida o vida armoniosa, que se mantiene desde los abuelos. Otros términos que se utilizan dentro el Pueblos Guarania, son Ivi Maraei, tierra sin mal, Iyambae: sin dueño, libre, pero que vive bajo las reglas de la comunidad (MDP, 2007: 16).

Quechua (Bolivia, Peru, Ecuador): Sumak Kawsay: que propone medidas de equilibrio y complementariedad entre los seres humanos tales como la minga, el randi-randi, el cambia-mano y la integración con la Pacha Mama, la naturaleza tan violentamente agredida por los capitalistas, que anteponen el lucro individual sobre cualquier otro elemento.

\subsection{Vivir bien, una aproximación al concepto}

Esta propuesta que viene de las comunidades, pueblos y de cada indígena - El Vivir Bien/ Buen Vivir- implica un crecimiento de la calidad de vida de la persona, pero en directa vinculación con la naturaleza, una búsqueda del equilibrio entre ser humano y naturaleza, que busca un bien común y no sólo crecimiento económico (Rojas, 2009: 1), fortaleciendo la cultura y sus identidades. Esto refleja un principio básico de la vida de los pueblos indígenas y su entorno. Al romperse esos equilibrios tenemos como consecuencia el cambio climático y desequilibrios que no son naturales, sino provocados por el ser Humano. Este crecimiento en calidad de vida se debe realizar a partir del sistema económico de los pueblos indígenas, sistema que les ha permitido subsistir por generaciones, sin alterar el equilibrio natural de la madre tierra.

El Vivir Bien expresa el encuentro entre pueblos y comunidades, respeta la diversidad e identidad cultural. Significa "Vivir Bien entre nosotros", es una convivencia comunitaria con interculturalidad y sin asimetrías de poder, "no se

${ }^{5}$ Provisto por Samuel Carpintero, abogado Ngobe. 
puede Vivir Bien si los demás viven mal". Se trata de vivir como parte de la comunidad, con protección de ella, en armonía con la naturaleza, "vivir en equilibrio con lo que nos rodea". También significa "Vivir Bien contigo y conmigo", que es diferente del "vivir mejor" occidental, que es individual, separado de los demás e inclusive a expensas de los demás y separado de la naturaleza.

El Vivir Bien es la expresión cultural que condensa la forma de entender la satisfacción compartida de las necesidades humanas, más allá del ámbito de lo material y económico. A diferencia del concepto occidental de "bienestar", que está limitado al acceso y a la acumulación de bienes materiales, incluye la afectividad, el reconocimiento y prestigio social. También es una práctica relacionada con la dignidad, la independencia y la singularidad, con las lenguas nativas y el bilingüismo, y con dimensiones inmateriales y subjetivas, como el aprecio y reconocimiento comunitario, el afecto y el ocio traducido en la fiesta. El Vivir Bien es la demanda de humanización del desarrollo donde la diversidad cultural asegura la responsabilidad y obligación social en el desempeño de la gestión pública. De esta manera, los cambios que existen dentro de la vida personal y comunitaria "el desarrollo" se convierten en un proceso colectivo de decisión y acción de la sociedad como sujeto activo y no como receptora de directivas verticales.

Resumiendo y rescatando algunas reflexiones sobre el Vivir Bien, es una propuesta que viene desde los Pueblos Indígenas de los cinco continentes, y que ha hecho eco en dos constituciones de América Latina, como es Bolivia y Ecuador; donde en el caso de Bolivia es la base de movimiento global contra el consumismo, la depredación de los recursos naturales y los actuales modelos de desarrollo.

En concordancia con el movimiento indígena, que a nivel internacional está realizando un esfuerzo para colocar en las agendas de discusión sobre el desarrollo, señalamos que el Vivir Bien no es lo mismo que el Vivir mejor, porque el objetivo final no es la acumulación de bienes materiales a costa del medio ambiente, sino contar con todas las necesidades básicas y que la naturaleza cumpla su parte como proveedor de los recursos de manera permanente, siempre y cuando se logre un equilibrio entre el aprovechamiento y la conservación. Por otro lado, la comprensión del vivir bien es dinámica, como dinámica es la vida de los pueblos indígenas; y donde la propuesta incorpora la concepción tradicional de los pueblos indígenas combinándolo con el movimiento ambiental y anticapitalista que defiende el planeta.

En marco de lo anterior, el Vivir Bien está basado en tres principios (i) complementariedad; (ii) reciprocidad; y (iii) valores y principios. Y cuenta con cinco pilares (i) armonía con la naturaleza, (ii) recuperación de la identidad, (iii) vivir 
en comunidad, (iv) espiritualidad y entretenimiento; y (iv) el contar con las necesidades básicas (solo lo que se necesita, no a la acumulación).

Partiendo de esta base, el Vivir Bien implica "el acceso y disfrute de los bienes materiales en armonía con la naturaleza, son relaciones para el disfrute, pero también es la parte humana, la realización afectiva, subjetiva y espiritual de las personas y la relación con los demás; la mujer y el hombre no están solos, aislados, viven primero en familia y luego en comunidad, el hombre y la mujer se siente bien en la medida que todos están bien", y postula una visión cosmocéntrica y diversidad cultural, es una expresión cultural que condensa la forma de entender la satisfacción compartida de las necesidades humanas, más allá del ámbito de lo material y económico. El Vivir Bien es también la satisfacción de la participación social, política y económica de las personas".

\section{BIBLIOGRAFÍA}

BANCO INTERAMERICANO DE DESARROLLO (2006). Estrategia para Pueblos Indígenas. Washington DC.

BONFIL, G. (1995). Etnodesarrollo: sus premisas jurídicas, políticas y de organización. En obras escogidas de Guillermo Bonfil Batalla. Tomo 2 (pp 464-480). INAH/INI, México.

ECOSOC/ONU (2010). Informe sobre el noveno período de sesiones (19 a 30 de abril de 2010). Documentos Oficiales E/2010/43, 2010 Suplemento núm. 23.

Dirección General de Planificación y Evaluación de Políticas para el Desarrollo/ AECID (2006). Estrategia Sectorial de la Cooperación Española con los Pueblos Indígenas. Madrid. España.

DEPARTAMENT OF ECONOMIC AND SOCIAL AFFAIRS, United Nations. (2010). State of The World's Indigenous Peoples. New York.

DEL POPOLO, F, OYARCE, A.M. y RIBOTTA, B. (2007). Indígenas urbanos en América Latina: algunos resultados censales y su relación con los Objetivos de Desarrollo del Milenio Notas de población $\mathrm{N}^{\circ} 86$ • C E PA L. p. 106

IWGIA (2010). The Indigenous World 2010.Copenhagen.

HUANACUNI, F. (2010). Buen Vivir / Vivir Bien Filosofía, politicas, estrategias y experiencias regionales andinas. Coordinadora Andina de Organizaciones Indígenas.

Ministerio de Planificación del Desarrollo (2009). Memoria taller Pueblos GuaraniProyecto Construcción de Indicadores del Vivir Bien, 1-9

MARTINEZ, J. (1986/7). Study of the problem of discrimination against indigenous populations. UN Doc. E/CN.4/Sub.2/1986/7 and 1-4.

Ministerio de Asuntos Indígenas y Pueblos Originarios (1998). Desarrollo con IdentidadPolítica Nacional Indígena y Originaria.

PÉREZ DE CUELLAR, J., ARIZPE, L., FALL, Y. et al. (1996), Nuestra diversidad creativa. Informe de la Comisión Mundial de Desarrollo y Cultura. UNESCO, México. 
ROJAS, D. (2009). El Buen Vivir: La Visión de Desarrollo de los Pueblos Indígenas en Centroamérica. Doc. In. I Encuentro Iberoamericano: Autonomía, Desarrollo con Identidad y Derechos de los Pueblos Indígenas. Madrid 9 y 10 de junio 09.

SEN A. (1987). Commodities and capabilities, Oxford University Press, Oxford India. Paperbacks, Nueva Delhi.

UNPFII. (2006). UN Economic and Social Council, Permanent Forum on Indigenous Issues, Fifth Session. "Action Programme for Second Indigenous Decade Launched." 15 May 2006.

http://www.un.org/esa/socdev/unpfii/en/news.html (Febrero, 24 del 2011). 